\title{
PROJEKTMANAGEMENT AM KONSTRUKTIONSBEISPIEL VANDALISMUSGESCHÜTZTER LEUCHTENSYSTEME
}

\author{
Friedhelm Pracht \\ Falkenweg 5, 35232 Dautphetal-Buchenau, Deutschland \\ E-mail:friedhelm.pracht@pracht-lichttechnik.de \\ Empfangen 12 April 2005; angenommen 15 September 2005
}

\begin{abstract}
Auszug. Gebäude im öffentlichen Bereich sowie städtische Verkehrsanlagen werden bedauerlicherweise durch Vandalismus beschädigt. Die selbe Erscheinung haben wir mit Beleuchtungskörper in den genannten Einsatzgebieten. Leuchten werden nicht nur der Witterung sondern auch dem zunehmenden Vandalismus ausgesetzt. Im Sinne der Produktverantwortung und des späteren Recyclings, nämlich mit möglichst wenig unterschiedlichen und leicht zu entsorgenden umweltfreundlichen Werkstoffen zu arbeiten, wird das Thema im Hinblick auf die Realisierung eines neuen vandalismusgeschützten Leuchtensystems in hoher Schutzart IP 65 und wahlweise in Schutzklasse I und II (schutzisoliert) behandelt.
\end{abstract}

Schlüsselwörter: Schlagfestigkeit, Langlebigkeit, Recycelbarkeit, Projektmanagement, Projektplanung, Produktverantwortung, Witterungsbeständigkeit, Vandalismus, Einfache Wartung, Rissbildung, Zugfestigkeit.

\section{PROJECT MANAGEMENT WITH THE CONSTRUCTIONAL EXAMPLE OF VANDAL-RESISTANT LUMINAIRE SYSTEMS}

\author{
Friedhelm Pracht
}

Falkenweg 5, 35232 Dautphetal-Buchenau, Germany

E-mail: friedhelm.pracht@pracht-lichttechnik.de

Received 12 April 2005; accepted 15 September 2005

\begin{abstract}
Unfortunately public buildings and municipal transportation facilities are vandalised. We have the same problem with luminaires in the mentioned application areas. Luminaires are not only exposed to weather but also to increasing vandalism. For the purpose of the product responsibility and the subsequent recycling, namely to work with as few different non-polluting and easy to depollute materials as possible, this issue is discussed with regard to the realisation of a new vandal-resistant liminaire system with high safety classification IP 65 and optionally in protection class I and II (double insulated).
\end{abstract}

Keywords: impact resistance, longevity, recycling, project management, project planning, product responsibility, weathering resistance, vandalism, easy maintenance, cracking, tensile strength.

\section{Einführung}

Der Begriff Vandalismus ist verständlicherweise negativ besetzt, in vielen Städten leider zur Normalität geworden.

Betroffen sind nicht nur Beleuchtungseinrichtungen, sondern auch andere gemeinnützige Anlagen, die aus Steuermitteln finanziert werden.
Das Forschungsvorhaben betrifft eine Leuchtenausführung speziell für diesen Bereich, insbesondere für die Beleuchtung in Fußgängerunterführungen, Bahnhöfen, Aufzügen, Treppen- und Parkhäusern sowie Brücken. Ein weiterer wichtiger Einsatzbereich der Neuentwicklung ist die Montage in Gefängnissen und geschlossenen Anstalten. 
Als Leuchtmittel dienen Leuchtstofflampen, die besonders geschützt, in einem schlagfestem Gehäuse eingebaut sind.

Die Lichtaustrittsfläche besteht aus einem klaren blendfrei, prismierten und schlagfestem Polycarbonat. Als Werkstoff für das Leuchtengehäuses wird vorzugsweise Aluminium verwendet.

Das Endprodukt ist als Schutzrecht angemeldet.

\section{Konstruktionsplanung nach einem Projektmanage- mentsystem}

Unter Berücksichtigung des Projektmanagementsystems wird der gesamte Fragenkatalog und ein Entwicklungsplan vorgelegt.

Alle Bauteile wurden im CAD-Verfahren konstruiert die Nullserie im Rapid Prototyping [1] - Rapid Product Development [2] realisiert.

\subsection{Strategische Ziele}

Der Einführung [3] von Projektmanagement sollte eine klare und eindeutige Strategie zugrunde liegen, denn würde man nur den aktuellen Trends folgen, käme es früher oder später zu schmerzlichen Rückschlägen.

Die Projektplanung im Rahmen des Projektmanagements soll den Aufwand ermitteln, der notwendig ist, um das Projekt durchzuführen [3]. Da der Umfang und die Vielschichtigkeit von Projekten zunehmen, gewinnt auch die Projektplanung immer mehr an Bedeutung.

\subsection{Ziele unter Beachtung der Produktverant- wortung}

Nachstehend wird eine Produktlinie behandelt, die in einem neuen Marktsegment vertrieben werden soll.

Für bisherige Entwicklungen aus dem Produktionsprogramm Leuchten höherer Schutzart wurden ausschließlich Kunststoffe oder Kunststoffverbundwerkstoffe eingesetzt.

Ziele nach $\S 22$ Abs. 1 des Kreislaufwirtschafts- und Abfallgesetzes [4] sind:

- Langlebigkeit bei Vandalismus

- Lichttechnische Effizienz durch eingebaute Hochleistungs-Spiegelreflektoren

- einfaches recyceln unter Beachtung der Warenrücknahmeverordung, WEEE (Waste of Electrical and Electronic Equipment gültig ab 13.08.2005)

- Im Projektplan ist weiterhin vorgesehen, dass möglichst wenig unterschiedliche Werkstoffe in der Konstruktion der Bauteile verwendet werden.

\subsection{Prozessbeeinflussung des Projektmanagements}

Der Entschluss zur Einführung des Projektmanagements in ein Unternehmen bringt erhebliche organisatorische Veränderungen mit sich, weshalb äußerst wichtig ist, sorgfältig zu planen und vor allem einer übergeordneten Strategie zu folgen.
Sie könnte exemplarisch wie folgendermaßen definiert sein [3]:

- größere Effizienz durch Zieleinhaltung

- bessere Mitarbeitermotivation z.B. durch projektorientierte Vergabe von Aufgaben, Zuständigkeiten und Vollmachten an motivierte Mitarbeiter

- größere Kundennähe durch eine stärkere Beachtung von Kundenwünschen und eine raschere Reaktion auf entsprechende Äußerungen. Image-Steigerung

- frühzeitige Integration und Abklärung bzw. Erfüllung der Normvorschriften

\section{Warum Projektmanagement?}

Kein Projekt ist zu klein, um nicht ernst genommen zu werden [3].

Vier wichtige Schritte stehen in engem Zusammenhang [3]:

- Definition

- Planung

- Realisierung

- Abschluss

Das Forschungsvorhaben wurde unter dieser Voraussetzung in folgenden Schritten durchgeführt [3]:

1. Was soll erreicht werden?

2. Wie ist der Stand der Technik?

3. Optimierung der Lösungswege

4. Konstruktive und mögliche Lösungen und Auswahl der geeigneten Werkstoffe

5. Fazit

Projektmanager oder Produktmanager kommen oft bei zeitlich befristeter Aufgabenstellung zum Einsatz. Für diese spezielle Aufgabe wurden ihnen besondere Verantwortlichkeiten übertragen.

Ein Produktmanager oder Produktkoordinator ist quer durch alle Funktionen für alle Maßnahmen seines betreuten Produktes zuständig.

Vorteile des Projektkoordinators sind [5]:

- die ausschließliche Beschäftigung mit dem wichtigen Projekt

- bessere Möglichkeiten zur Koordination

- große Flexibilität und Reaktionsfähigkeit mögliche Nachteile sind:

- Gefahr von Konflikten durch Mehrfachunterstellung ( Projekt, Stab, Linie )

- Schwierigkeiten bei der Durchsetzung der Projektinteressen

- Starke Abhängigkeit des Projekterfolges von den Fähigkeiten von einzelnen Personen

\section{Projektplan Vandalismusleuchte}

Der detaillierte Projektplan ist folgendermaßen aufgebaut: 


\section{VORPLANUNG - Zusammenstellung der Informa- tionen}

- Marktanfrage und Informationen aus dem Vertrieb über die vorhandenen Marktlücken, Wettbewerbsbetrachtung, Marktchancen etc., an die Geschäftsleitung

- Einberufung einer Brainstorming Sitzung durch die GF mit - Vertrieb - Produktion -Konstruktion/TL

- Entscheidung und Freigabe des Projektes durch die GF

- Bestimmung der Verantwortlichkeiten und Festlegung des Projektleiters

- Erstellung eines Fragenkataloges (Pflichtenheft) durch Vertrieb und Projektleiter/ung

- Prüfung der technischen Machbarkeit durch TL,

Nach der Entscheidung und Freigabe und Klärung der technischen Machbarkeit erfolgt die Hauptplanung des Projektes

HAUPTPLANUNG - Haupttechnologie

- Terminplanfestlegung durch Projektleiter

- Festlegung der Verantwortungsbereiche (en detail )

- interner Auftrag für Lösungsvarianten auf der Grundlage des vorhandenen Fragenkataloges

- Patentrecherche und Stand der Technik

- Normenabklärung

- Terminvorgabe, evt. Terminkorrektur - Diskussion der ersten Konstruktionsvorschläge

- Auswahl und Prüfung einer Lösungsvariante

- Interdisziplinäre Erfahrungen und Klärung aller Detailfragen

- QS-Einbindung und Klärung der Prozesstechnik

- Festlegung der endgültigen Konstruktionslösung incl. optischer Betrachtung

- Endgültige Konstruktion der Bauteile

- Werkstoffdiskussion und Materialfestlegung

- Erstellen der Stücklisten

- Anfragestart des Einkaufes, EK

- Prüfung der Angebote, Vorkalkulation

- Terminüberwachung und Klärung aller noch offenen Fragen mit Konstruktion - TL - KL - Vertrieb QS - Produktion

- Fertigungsfreigabe der Prototypen durch TL

ENDPLANUNG - Produktrealisierung

- Erstellung der Stereolithografieteile

- Komplettbau eines Prototypes

- Übergabe im VDE-Prüfinstitut zur Abklärung aller Normenfragen

- Letzte Abstimmung in einer gemeinsamen Sitzung mit GF, KL, VT, QS, GF: Geschäftsleitung, KL:Kaufmännische Leitung,VT:Vertriebsleitung, QS:Qualitätssicherung

- Wirtschaftlichkeitsanalyse

- Kalkulationserstellung

- Zielpreisfestlegung

- Klärung aller Detailfragen, Festlegung der Lieferanten, gemeinsam EK und TL
- Terminplanerstellung bis zur Serienfertigung

- Schutzrechtsanmeldung

- Freigabe der Werkzeugaufträge

PRODUKTIONSPLANUNG

- Fertigung der Nullserie

- Erste Messungen am Endprodukt, Prüfungen

- Abstimmung und Detailfragen der Prozesstechnik (Fertigungstechnik, Werkzeuge, Verpackungsart, EK-Einbindung etc.)

- Abschluss der Prüfungen und Erteilung der Zertifikate

- Fertigungsfreigabe durch die TL

- QS-Maßnahmen und Dokumentationen

MARKTEINFÜHRUNG

- Marketingmaßnahmen, Prospekterstellung, Verkaufsaktionen, Kundenschulungen etc.

- Endgültige Bruttopreisfestlegung, Rabattvorgaben

- Vertriebsinformationen an die ADM's

- Terminfestlegung für Verkaufsstart

- Beginn der Produktion

- Markteinführung

Der Projektplan kann wegen unterschiedlicher Komplexität durchaus von anderen Produktlinien abweichen.

Als Basis einer Produktentwicklung folgt beispielhaft ein Flussdiagramm Abb. 1 und Abb. 2. Hier wird detailliert der gesamte Entwicklungsablauf gezeigt.

\subsection{Flussdiagramm eines Entwicklungsauftrages}

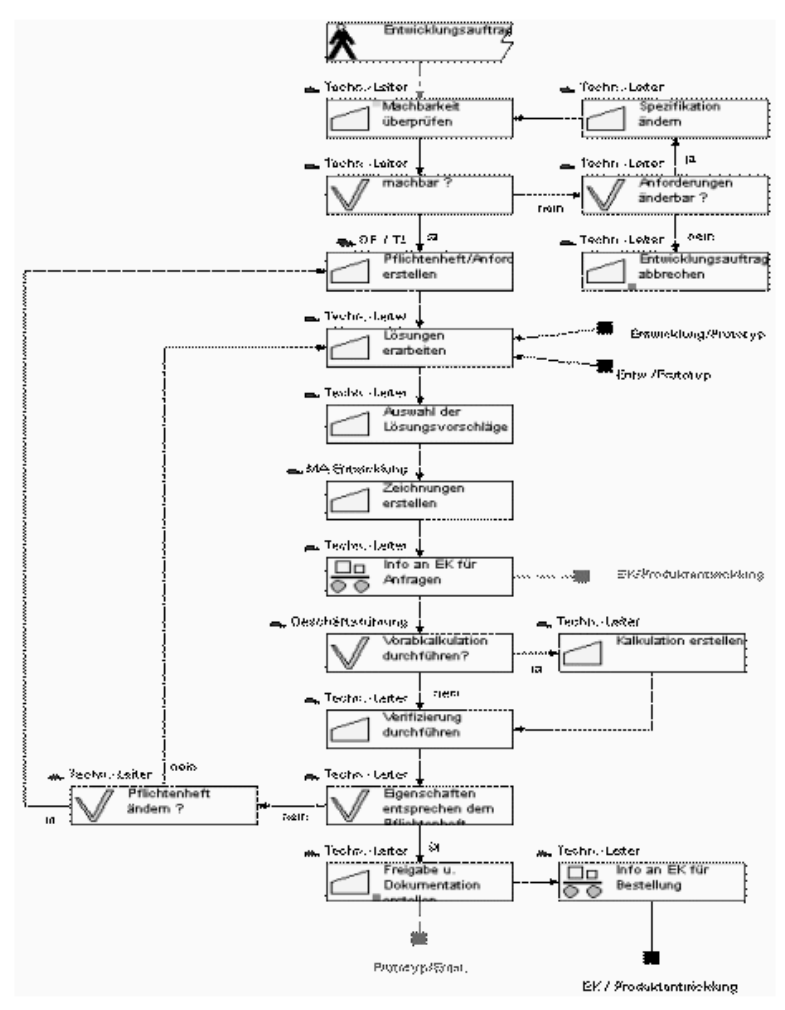

Abb. 1. Beginn des Ablaufes einer Produktentwicklung

Fig 1. Start of the course of action of the development of a product 


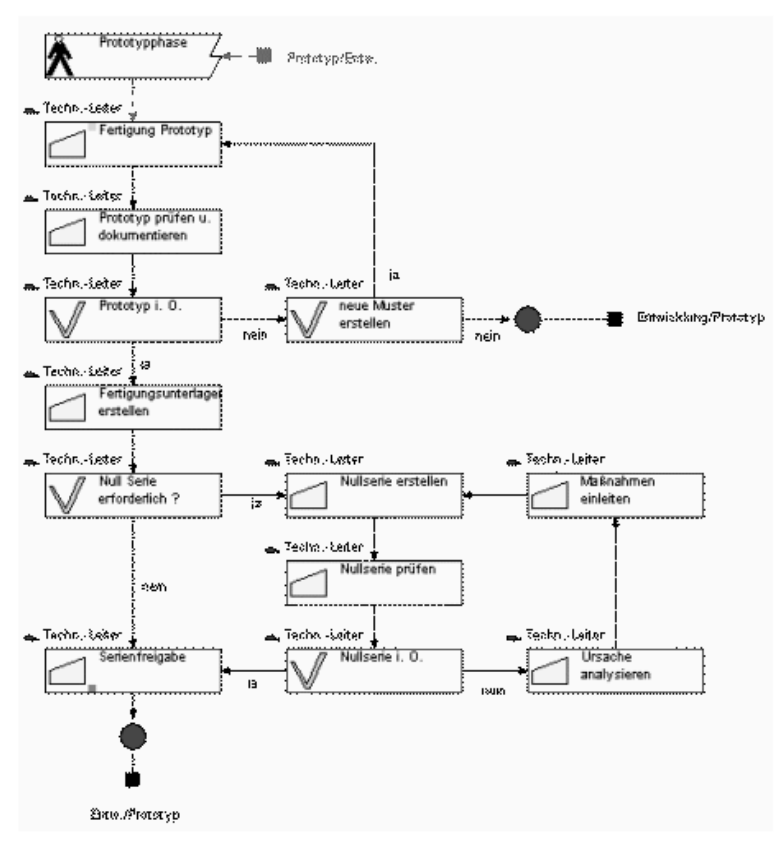

Abb. 2. Prototyp und Null - Serie

Fig 2. Prototype and pilot run

\subsection{Was soll erreicht werden?}

Aufgabe der Neukonstruktion ist ein formschönes, aber weitgehend gegen Vandalismus geschütztes Produkt zu entwickeln. Im Sinne der Produktverantwortung nach $\S 22$ des Kreislaufwirtschafts- und Abfallgesetzes sollten möglichst wenige verschiedene, und leicht recycelbare Werkstoffe Verwendung finden. Ein besonderer Aspekt ist in diesem Fall die Langlebigkeit des Produktes in vandalismusgefährdeten Einsatzgebieten.

Eine weitere Zielvorgabe ist, eine Konstruktionslösung zu finden, die durch intelligente Weiterentwicklungen, mit deutlichen, technischen Unterscheidungsmerkmalen, zum heutigen Stand der Technik, dem späteren Anwender optimale Problemlösungen unter ökonomischer und ökologischer Betrachtung bieten.

Zu Beginn der Überlegungen waren wichtige Grundsatzfragen zu klären. Die Antworten sind entscheidend für die Qualität der Problemlösungen für das späteren Endprodukt.

Zum Verständnis werden einige Fragen genannt, die im Vorfeld diskutiert wurden:

- In welchem Winkel sollte die Lichtaustrittsfläche sein?

- Welche Menschen betreiben Vandalismus?

- Sind sie groß gewachsen, besonders kräftig, und wie setzen sie ihre Übergriffe ein?

- Ist bei den Menschen auch Diebstahl oder nur Zerstörung Ziel?

- Zu welcher Tageszeit erfolgen die Beschädigungen?

- Wie fest muss die Abdeckung sein, Kerbwirkung,
Schlagwirkung in welche Richtung?

- Wo werden die Leuchten montiert? Sind es Einzelleuchten, Eckleuchten etc.?

- Wie wichtig ist die ästhetische Form des Endproduktes?

- Wird Vandalismus mit gefährlichen Hilfs- oder Spezialwerkzeugen ausgeübt?

Die Auswahl der Fragen wurden aufgrund langjähriger Erfahrung gestellt. Ergebnis und Kriterien sind aus den intensiven Gesprächen mit den planenden Ingenieuren des Gebäudemanagements und den Wartungsabteilungen des späteren Betreibers entstanden. z.B. Erfahrungen der Wartungsabteilung Stadtbeleuchtung

\subsection{Stand der Technik}

Um spätere Schutzrechts- und Geschmacksmusterverletzungen auszuschließen, ist der Stand der Technik zu recherchieren.

Es gibt von namhaften Herstellern optisch und haptisch recht unterschiedliche Lösungen, wobei die Leuchtengehäuse neben rostfreiem Stahl auch aus Aluminium oder Zinkdruckgusslegierungen bestehen.

Als Material für die Lampenabdeckungen wird vorzugsweise nur ein Polycarbonat verwendet.

Die Hauptunterschiede liegen im optischen Bereich, besonders in der Form des Querschnitts der Leuchten.

Erhebliche technische Unterschiede gibt es bei der Art der Montage der Lampenabdeckung und der Befestigung der Leuchtengrundgehäuse.

Die Befestigung der Leuchtengehäuse ist meist auf Fixmasse beschränkt.

\section{Konstruktiver Lösungsweg}

Nach dem in Kapitel 4 in der Vorplanung festgelegten Fragenkatalog/Pflichtenheft, siehe auch in Abb 1, Flussdiagramm, werden in Kapitel 5 die konstruktiven Lösungswege und Kriterien diskutiert und entwickelt.

Die Neukonstruktion betrifft eine Leuchte mit einem decken- oder wandseitig befestigbaren Grundprofil, das stirnseitig jeweils mit einem den Leuchtenquerschnitt weitgehend angepassten Kopfteil verbunden ist, das der Aufnahme von Funktionselementen dient, andererseits jeweils mit einem formmäßig angepassten Druckdeckel verschraubbar ist, wobei zwischen den beiden endseitigen Druckdeckeln eine lichtdurchlässige Lampenabdeckung gehaltert ist.

Leuchten dieser Art, bei denen als Leuchtmittel im Regelfall eine oder mehrere beidendig gesockelte und auch einseitig gesockelte Leuchtstofflampen verwendet werden, sind in vielfältigen Ausführungsformen bekannt und werden vor allem auch zur Beleuchtung öffentlicher Räume, wie z.B. in U-Bahnhöfen, Unterführungen, Einkaufspassagen 
in Untergeschossen und dergleichen, eingesetzt.

In öffentlich zugänglichen Bereichen kommt es immer wieder zu Vandalismus, d.h. die Leuchten werden nicht nur mutwillig beschädigt, sondern häufig auch entwendet. Dies führt zu beträchtlichen Schäden die zu Lasten der Allgemeinheit gehen.

Dies sollte nach Möglichkeit verhindert werden.

Aufgabe der Neuentwicklung ist es daher, eine gegen Vandalismus geschützte Leuchte zu entwickeln d.h. sie so auszugestalten, dass ihre Entwendung verhindert oder zumindest so erschwert wird, dass der zum Diebstahl erforderliche Zeitaufwand abschreckend groß ist.

Diese Aufgabe wird ausgehend von der eingangs genannten Leuchte nach der Neukonstruktion im Wesentlichen dadurch gelöst, dass zumindest zwei zur Verschraubung mit Wand oder Decke bestimmte, sich über die Breite des Grundprofils erstreckende Klammerelemente vorgesehen sind, die die Seitenränder des Grundprofils übergreifen und mit ihm federnd verrastbar sind, weshalb für die Lampenabdeckung seitlich begrenzende und zwischen den Druckdeckeln gehalterte Schutzprofilteile vorgesehen sind, die nach der Verschraubung der Druckdeckel mit den Kopfteilen die Verrastungsbereiche zwischen den Klammerelementen und dem Grundprofil abdecken und von außen unzugänglich machen.

Durch diese wichtigen konstruktiven Maßnahmen und insbesondere durch die Ausgestaltung und Lage der speziellen Schutzprofilteile wird erreicht, dass zum einen eine einfache Montage, durch variable Befestigungsabstände, der Leuchte gewährleistet ist und zum anderen nach erfolgter Montage die zur Wand- oder Deckenbefestigung verwendeten Elemente nicht mehr sichtbar und vor allem auch von außen nicht mehr zugänglich sind, so dass ein Herausreißen der Leuchte aus der Halterung bzw. das rasche Entwenden der montierten Leuchte praktisch

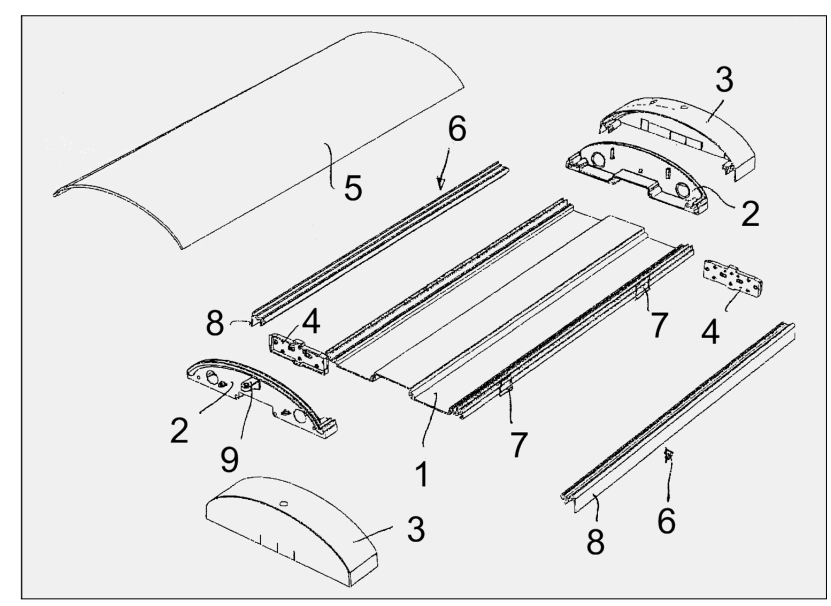

Abb. 3. Explosionsdarstellung der wesentlichen Komponenten der neuen Leuchte

Fig 3. Exploded view of the fundamental parts of the new luminaire nicht mehr möglich ist.

Vorzugsweise sind die seitlichen Schutzprofilteile mit den Druckdeckeln form- und/oder kraftschlüssig verbunden, so dass insgesamt eine stabile Rahmeneinheit vorliegt, wobei die Schutzprofilteile gleichzeitig Blockierelemente gegen ein Herausschwenken der Klammerelemente aus ihrer Verrastung mit dem Grundprofil bilden, d.h. es wird auf diese Weise sichergestellt, dass jegliche Manipulationsversuche zum Lösen der Klammerverbindung erfolglos bleiben.

Weitere Vorteile der Neuentwicklung sind in den Optimierungspunkten angegeben und werden auch anhand der Erläuterung eines Ausführungsbeispiels unter Bezugnahme auf die Zeichnung beschrieben.

\section{Bezugszeichenliste}

1. Grundprofil

2. Kopfteile

3. Druckdeckel

4. Träger für Funktionsleuchte

5. Lampenabdeckung transparent

6. Schutzprofilteile

7. Klammerelemente

8. Abdeckschenkel

9. Schraubaufnahmen

Abb. 3 zeigt die Grundkomponenten der Leuchte, nämlich ein einteiliges, langgestrecktes Grundprofil (1), stirnseitig mit dem Grundprofil fest zu verbindende Kopfteile (2), eine lichtdurchlässige Lampenabdeckung (5), die mit zwei Druckdeckeln (3) und randseitig anzubringenden Schutzprofilteilen (6) zu einer Einheit zusammen gefügt werden kann. An den Kopfteilen (2) zu befestigende Trägerteile (4) zur Anbringung von Funktionselementen, wie z.B. Fassungen für Leuchtstofflampen und dergleichen, sind ebenfalls dargestellt.

In Abb. 4 werden aus den Einzelkomponenten die

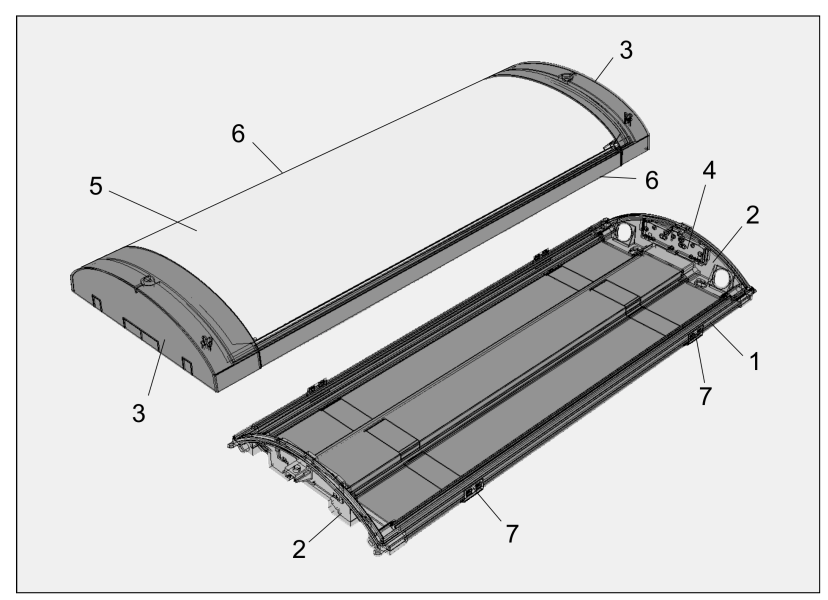

Abb. 4. Zwei Baueinheiten, bestehend aus den in Abb. gezeigten Einzelkomponenten, vor deren Zusammenfügung

Fig 4. Two constructional units made of single parts shown in Fig 3, before their junction 
gezeigten Baueinheiten gebildet, nämlich eine erste Baueinheit bestehend aus dem Grundprofil (1) und den stirnseitig mit dem Grundprofil (1) verbundenen Kopfteilen (2) mit zugehörigen Trägerteilen (4), sowie eine zweite, bestehend aus den beiden Druckdeckeln (3), den mit den ihnen (3) fest verbundenen Schutzprofilteilen (6) und der von diesen Komponenten gehaltenen und vorzugsweise mit diesen Komponenten dicht verbundenen Lampenabdeckung (5). Weitere Einzelheiten bedürfen keiner Beschreibung, da sie beliebig gewählt werden können und für den Gegenstand der vorliegenden Neuentwicklung ohne besondere Bedeutung sind.

Zur Montage dieser neuen Leuchte sind die in Abb. sichtbaren Klammerelemente (7) erforderlich, die bevorzugt aus rostfreiem Federstahl hergestellt und mittels Schrauben an der Wand oder Decke zu befestigen sind.

Die Befestigungsabstände sind variabel und zeitsparend, durch das geschützte, diebstahl- und vandalismussichere Klammersystem aus rostfreiem Federstahl.

Nach erfolgter Montage der Klammerelemente (7) kann das Grundprofil 1 in die Klammerelemente eingeschnappt werden, d.h. es werden zwischen den Klammerelementen (7) und dem Grundprofil (1) Schnapp-Rast-Verbindungen gebildet, wobei die freien, nach oben gebogenen Schenkelenden der Klammerelemente (7) die profilierten Randbereiche des Grundprofils übergreifen und in eine entsprechende Profilausnehmung einschnappen, so dass das Grundprofil stabil in seiner vorgesehenen Position gehalten wird.

Auf dieses ortsfixierte Grundprofil mit den zugehörigen Kopfteilen (2) wird dann die in Abb. gezeigte Baueinheit mit der Lampenabdeckung (5) aufgesetzt und mit Schrauben fixiert, die in Schraubaufnahme (9) der Kopfteile eingreifen (Abb. 3). Wenn man die erforderlichen elektrischen Verdrahtungsarbeiten außer Acht lässt, dann ist die Montage der Leuchte mit der erfolgten Verschraubung der Druckdeckel (3) mit den Kopfteilen (2) beendet.

Für den durch die neue Konstruktion erbrachten Schutz gegen Vandalismus ist die Verwendung und Ausgestaltung der Schutzprofilteile (6) und deren Zusammenwirken mit den Schnapp-Rast-Verbindungen zwischen den Klammerelementen (7) und dem Grundprofil (1) von ganz entscheidender Bedeutung.

Als Schutzprofilteile (6) werden vorzugsweise stabile Profile, insbesondere aus Metall, verwendet, die über Formund/oder Kraftschlussverbindung mit den Druckdeckeln verbunden sind, so dass sich insgesamt eine sehr stabile Rahmenkonstruktion ergibt.

Die Schutzprofilteile (6) sind konstruktiv so gestaltet, dass sie einerseits abdichtend in ein entsprechendes randseitiges Gegenprofil des Grundprofils (1) eingreifen und andererseits einen Abdeckschenkel (8) aufweisen, der bezüglich der Klammerelemente (7) als Blockiereinheit wirkt und verhindert, dass diese Klammerelemente (7) aus ihrer Verrastungsposition herausgeschwenkt oder herausgedrückt werden können. Die Querschnittsgestaltung und Haptik der Schutzprofilteile ist entscheidet für eine sichere Abdichtung zum Grundprofil. Ein weiterer wichtiger Parameter ist die Formgebung und das Schenkelmaß über der abzudeckenden Befestigungsklammer. Dieser wichtige Konstruktionsteilaspekt ist entscheidend für eine diebstahlsichere Montage der Leuchte. Durch mehrfache Prototypenfertigung gerade dieses wichtigen Konstruktionselementes -Schutzprofilteil (6) konnte eine optimale Maßabstimmung erreicht werden. Die Leuchte kann selbs durch eine große Kraftaufwendung nicht von der Montagefläche ausgehebelt werden. Neben dem Schlagschutz der Leuchte ein wichtiger Faktor für ein optimales Produkt.

Dieser Abdeckschenkel (8) macht die Klammerelemente (7) zudem von außen her unzugänglich, d.h. bei montierten Leuchten sind die Klammerelemente (7) weder hinsichtlich ihrer Position noch hinsichtlich ihrer Funktion erkennbar. Wegen der mechanischen Verkopplung von Grundprofil (1) und Schutzprofilteilen (7) kann der Abdeckschenkel (8) praktisch auch nicht weggebogen werden, um Zugang zu den Klammerelementen (7) zu erhalten.

Die somit realisierte Mehrfachfunktion der Schutzprofilteile (6) gibt die nötige Sicherheit gegen Vandalismus bzw. Schutz vor Diebstahl, und zwar ohne jegliche Erhöhung des Montageaufwands.

\section{Konstruktive Optimierungspunkte}

Der konstruktive Lösungsweg wurde im vorgenannten Kapitel eingehend diskutiert. Die folgenden Punkte sind Optimierungen die sich als Nachteil bei den gebauten Stereolithographieteilen ergeben haben und ergeben deutliche Verbesserungen z.B. Optimierung der Schutzprofilteile (6).

1. Leuchte mit einem decken- oder wandseitig befestigbaren Grundprofil (1), das stirnseitig jeweils mit einem der Querschnittsform der Leuchte weitgehend angepassten Kopfteil (2) verbunden ist. Es dient einerseits zur Aufnahme von Funktionselementen und andererseits jeweils mit einem formmäßig angepassten Druckdeckel (3) verschraubar, (Spezialschraub-/ Verschlusslösungen die nur durch Sonderwerkzeuge lösbar sind, sind alternativ möglich ) wobei zwischen den beiden den endseitigen Druckdeckeln (3) eine lichtdurchlässige Lampenabdeckung (5) gehaltert ist.

Es sind zumindest zwei zur Verschraubung mit Wand oder Deckel bestimmte, sich über die Breite des Grundprofils (1) erstreckende Klammerelement (7) vorgesehen, die die Seitenränder des Grundprofils 
(1) übergreifend mit dem Grundprofil (1) federnd verrasten.

Für die Lampenabdeckung (5) sind seitlich begrenzende und zwischen den Druckdeckeln (3) gehalterte Schutzprofilteile (6) vorgesehen, die nach der Verschraubung der Druckdeckel (3) mit den Kopfteilen (2) die Verrastungsbereiche zwischen den Klammerelementen (7) und dem Grundprofil (1) abdecken und von außen unzugänglich machen.

2. Das Grundprofil (1) mit den endseitig angebrachten Kopfteilen (2) einerseits und die Lampenabdeckung (5) mit den beiden Druckdeckeln (3) sowie den seitlichen Schutzprofilteilen (6) andererseits bilden eine vormontierte Baueinheit.

3. Die seitlichen Schutzprofilteile (6) sind mit den Druckdeckeln (3) form- und/oder kraftschlüssig verbunden.

4. Die lichtdurchlässige Lampenabdeckung (5) ist sowohl mit den Druckdeckeln (3) als auch mit den seitlichen Schutzprofilteilen (6) verklebt.

Die Lampenabdeckung kann wahlweise im CoExtrusionsverfahren mit einem zusätzlichen Werkstoff im Bereich der Oberfläche in minimaler Wandstärke veredelt werden.

Die Schlagfestigkeit der Abdeckung wird dadurch nicht gemindert und Sprühfarben haften daher nicht.

5. Die Schutzprofilteile (6) sind mit dem Grundprofil (1) formschlüssig kuppelbar und haben einen außenliegenden Abdeckschenkel (8), der die Längsseite des Grundprofils (1) und die Verrastungsbereiche zwischen Grundprofil (1) und Klammerelementen (7) übergreift bzw. abdeckt.

6. Die vor allem mit dem Grundprofil (1) durch Formschlusskupplung verbundenen Schutzprofilteile (6) bilden Blockierelemente gegen das Ausschwenken der Klammerelemente (7) aus den Verrastungsposition mit dem Grundprofil (1).

\section{Werkstofflösungen für besondere Einsatzgebiete}

Die Leuchte kann in vandalismusgefährdeten Einsatzgebieten montiert werden.

Folgende Parameter wurden diskutiert, die nochmals aufgeführt werden sollen:

- Extrem flache und formschöne Optik

- Haptik (Anfassbarkeit) mit wenigen Angriffsmöglichkeiten

- Beständigkeit gegen Duktilität(Verformbarkeit) bei mutwilliger Beschädigung

- Hohe Effizienz durch einen innenliegenden Hochleistungsspiegel

- Geschützter Einsatz von magnetischen und elektronischen Betriebsgeräten in Schutzklasse I und

\section{Schutzklasse II ( schutzisoliert)}

- Einfache Wartung

- Langlebigkeit

- Einfaches Recycling unter der Prämisse, dass die zu recycelten Werkstoffe von den verantwortlichen Firmen wieder verwertet werden können

\subsection{Produktionsverfahren}

Die Produktionsverfahren sollen hier nicht behandelt werden, sie dienen rein informativ als Ergänzung.

Das Leuchtengehäuse wird im Strangpressverfahren, die Lampenabdeckung wird im Kunststoff-Co- oder im Extrusionsverfahren hergestellt.

Weitere Bauteile werden im Spritzgieß- bzw. im Druckgießverfahren produziert.

\subsection{Mögliche Werkstoffe}

Durch langjährige Erfahrungen in der Konstruktion und nach Rücksprache mit Materialherstellern hat sich wegen des Anforderungsprofiles eine Auswahl auf einige wenige Werkstoffe ergeben.

Dies sind:

1. Leuchtenbasisgehäuse: Aluminium

2. Lampenabdeckung: Polycarbonat, glasklar standard 2.1. Lampenabdeckung modifiziert, Graffitiresistent

3. Fassungsträger- und Leuchtenendgehäuse: Polyamid

4. Gehäuseenddeckel: Bei diesem wichtigen Teil werden in dem Artikel noch Varianten diskutiert: Aluminium/Legierungen, Zink-Druck-gusslegierung oder Magnesium bzw. eine Magnesiumlegierung

5. Hochglanz-Alu-Spiegel mit 99,99\% Reinheitsgehalt

6. Kleinteile und elektrische Bauteile, Draht etc.

Die Werkstoffauswahl der Position 1, 3, 5 und 6 sind unstrittig.

Für den wichtigen Gehäusedeckel bieten sich Varianten die nachstehend diskutiert werden.

Anforderungsprofil Gehäuseenddeckel:

- Hohe Schlagfestigkeit und geringe Rissbildung bei Beanspruchung

- gute Beschichtbarkeit und Haftung der Oberfläche durch nachträgliche resistente Vergütungen (Korrosionsschutz)

- Wetterbeständigkeit und Langlebigkeit

- Geringe Brennbarkeit

- geringes Gewicht

- Gute Verarbeitung

- Gute Recycelbarkeit

- Befestigung mit unverlierbaren Zylinderkopfschrauben mit besonderer Verschlussgeometrie aus rostfreiem Stahl, nur mit Spezialwerkzeug lösbar

\subsection{Aluminium Al}

Etwa $8 \%$ der Erdrinde besteht aus Aluminium, aber stets in chemischen Verbindungen mit anderen Elementen. 
Meist kommt es in Gesteinen wie Ton, Gneis, Granit und Basalt vor.

Der Einwand Kapitel 7.3., 7.4 und 7.5 von HERRN PROF. KLIBAVICIUS ist richtig.

Diese Informationen sind rein informativ als Erklärung für eine letztendliche Werkstoffwahl zu Aluminium hin.

Man kann sicher darauf verzichten, es ist aber auch kein

Fehler wenn zur Ergänzung diese Kapitel bleiben.

Meine höfliche Bitte : entscheiden Sie ob

Kapitel 7.3., 7.4. und 7.5 bleiben

\subsubsection{Technische Daten}

- Dichte $2,7 \mathrm{~g} / \mathrm{cm}^{3}$

- Schmelzpunkt: $660{ }^{\circ} \mathrm{C}$

- Gute thermische und elektrische Leitfähigkeit

- Aluminium hat eine geringe Zugfestigkeit, aber eine hohe Dehnbarkeit, ist sehr zäh, Härte und Verschleißwiederstand sind gering.

\subsubsection{Herstellung}

Bei der Gewinnung von Aluminium sind zwei Vorgänge zu unterscheiden:

- Die Gewinnung der Tonerde

- Die Reduktion zu Aluminium

Mit Hilfe von Natronlauge, Druck und Wärme, wird aus feingemahlenem, ausgewaschenen und anschließend getrocknetem Bauxit durch eine chemische Umsetzung und einem Glühprozess bei ca. $1.300^{\circ} \mathrm{C}$, die reine weiße Tonerde, also das Aluminiumoxyd gewonnen.

Diesen Vorgang nennt man auch Kalzinierung. Die Trennung von Aluminium und Sauerstoff erfolgt durch Schmelzelektrolyse. Nach Zusatz von Kryolith wird der Schmelzpunkt der Tonerde von $2000{ }^{\circ} \mathrm{C}$ etwa $950{ }^{\circ} \mathrm{C}$ herabgesetzt. Vor dem Vergießen dieser Flüssigkeit, lässt man das Aluminium von ca. $850^{\circ} \mathrm{C}$ bis knapp über den Schmelzpunkt abkühlen, so dass Fremdkörper, wie z.B. Tonerde- und Kryanolithstücken nach oben schwimmen, und leichter entfernen können.

Reinstaluminium $(99,99 \%)$ wird nach einer sog. Dreischichtelektrolyse hergestellt.

Als Ausgangsmaterial wird unreines Aluminium verwendet. Zur Herstellung von 1 Tonne Aluminium, sind 2 Tonnen Tonerde bzw. 4-5 Tonnen Bauxit erforderlich.

Es werden dabei etwa $550 \mathrm{~kg}$. Elektrodenkohle und $14000 \mathrm{kWh}$ elektrische Energie verbraucht.

Die Elektrolyseofen arbeiten mit 5-6 Volt Gleichspannung und 30 000-100 000 A. $40 \%$ des Aluminiumpreises sind deswegen Energiekosten (Angaben Aluminiumverband).

\subsubsection{Verarbeitung}

Aluminium kann im Strangpressverfahren und im Druckgießverfahren hervorragend verarbeitet werden.
- Gute Spanbarkeit und gut polierbar.

- Al-Gusslegierungen sind mit einem „,G“ gekennzeichnet.

- Oft sind die Legierungen mit Magnesium verschmolzen.

\subsubsection{Eigenschaften}

Eloxiertes Aluminium ist korrosionsbeständig gegenüber Seewasser. Wie schon erwähnt hat Al bei einer Dichte von $2,7 \mathrm{~g} / \mathrm{cm}^{3}$ nur ein Drittel von Stahl. Aluminiumlegierungen verfügen im Allgemeinen über eine Zugfestigkeit zwischen 70 und $700 \mathrm{MPa}$. Die Zugfestigkeit von Legierungen, die für das Strangpressen verwendet werden, bewegt sich zwischen 150-300 Mpa (Megapascal).

Druck Pa: $1 \mathrm{~Pa}=1 \mathrm{~N} / \mathrm{m}_{\llcorner}=0,01 \mathrm{mbar}$

Newton $\mathrm{N}: 1 \mathrm{MN}=10^{3} \mathrm{kN}=1.000 .000 \mathrm{~N}$

$1 \mathrm{~N} / \mathrm{mm}_{\iota}=10 \mathrm{bar}=1 \mathrm{MN} / \mathrm{m}_{\iota}=1 \mathrm{Mpa}$

KANN ENTFERNT WERDEN.

Im Unterschied zu den meisten Stahlsorten tendiert Aluminium bei niedrigen Temperaturen nicht zur Brüchigkeit. Bei hohen Temperaturen hingegen lässt die Festigkeit nach.

Im Vergleich zu anderen Metallen verfügt Al über einen relativ großen Längenausdehnungskoeffizienten, was konstruktiv zu berücksichtigen ist, und in Punkt 8.3. diskutiert und berechnet wird.

Aluminium reflektiert sichtbares Licht und Strahlungswärme ausgezeichnet. Das gleiche gilt für dichte Aluminiumbehälter für gute EMV (Elektromagnetische Verträglichkeit) Abschirmqualitäten.

Al reagiert mit Luftsauerstoff. Es bildet sich eine ganz natürliche dünne Oxidschicht die trotzdem einen hervorragenden Korrosionsschutz bietet. Bei Beschädigung regeneriert sich diese Schicht selbst.

Durch eine anodische Oxidation, das Eloxieren, erhält die Al-Oberfläche eine dickere Oxidschicht, die Abriebfestigkeit und Korrosionsschutz erheblich verbessern. Die Schutzwirkung geht jedoch verloren, wenn die Oxidschicht durch saure oder alkalische Einwirkung chemisch angegriffen und aufgelöst wird. Der chemisch neutrale Bereich des guten Korrosionsverhaltens beschränkt sich auf pH 5- 8.

\subsubsection{Recycling}

Kaum ein nicht eisenhaltiges Metall lässt sich so leicht verwerten wie Aluminium. Dank seiner physikalischchemischen Eigenschaften hilft es außerdem Energie zu sparen.

\section{Warum?}

Dies liegt vor allem daran, dass einmal hergestelltes Aluminium die Energie gespeichert hat, die zur elektronischen Umwandlung des Rohstoffes Aluminiumoxyd aufgewendet werden musste [6].

Bei keinem anderen Metall ist die Energiebilanz bei der Wiederverwertung deshalb so positiv. 
In einem Tagungsvortrag des Alu-Verbandes der Schweiz in 2002 wurden folgende wichtige Erfahrungen veröffentlicht [6]:

- Aluminium kann immer und immer wieder rezykliert werden.

- Aluminiumschrott ist ein Wirtschaftsgut mit sehr hohem Werterhaltungsgrad

- Das Aluminium-Recycling ist eine traditionelle industrielle Tätigkeit, deren Produktionsprozesse laufend optimiert werden.

- Die wesentlichen kommerziellen und technischen Parameter für das Recycling von Aluminium werden durch zweckmäßige Logistikkonzepte sowie Separations- und Aufbereitungsverfahren vor dem eigentlichen Umschmelzen gesetzt.

- Die verschiedenen dabei anfallenden Fremd- und Restsstoffe können einerseits durch geeignete Verfahren wieder eingesetzt bzw. als Wertstoffe verkauft werden oder andererseits soweit wie möglich umweltgerecht versorgt werden

- Beim Rezyklieren von Aluminium wird bis zu $95 \%$ der bei der Erstproduktion eingesetzten Energie eingespart, d.h. die Sekundärenergie beträgt nur 5 $\%$ der Primärenergie

- Aluminium ist eine Energiebank. Die Herstellungsenergie bleibt beim Recycling erhalten

- Weil Aluminium rezyklierbar ist, muss nicht für alle Anwendungsmöglichkeiten Hüttenmetall verwendet werden. Dies stellt eine bedeutende Schonung der Ressourcen dar.

- Rezykliertes Al wird für alle technischen Anwendungsgebiete benutzt . Infolgedessen müssen keine speziellen Verwendungszwecke und Märkte gesucht werden.

- Durch diesen Nachfragesog liegen die Recyclingraten bei den wichtigsten Aluminium-Anwendungen bei über $80 \%$

Man unterscheidet beim Recycling (Abb. 5) [7].

Mit Primäraluminium allein kommt die Wirtschaft weltweit schon lange nicht mehr aus.

Der Al-Bedarf in Europa lag 1998 bei 7.213.000 Tonnen und wird im Jahr 2020 einen geschätzten Wert von

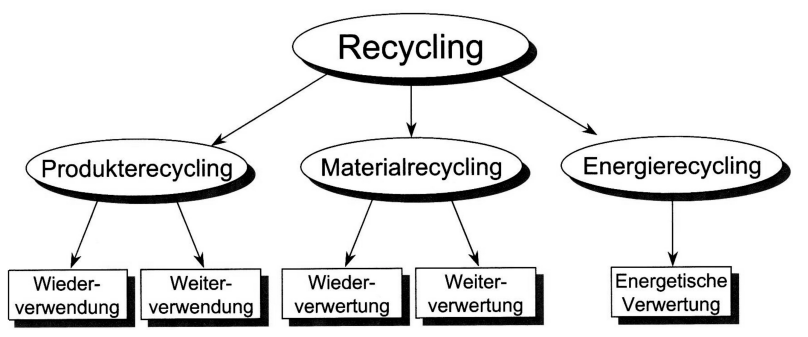

Abb. 5. Recycling

Fig 5. Recycling
18.000.000 Tonnen erreichen.

1998 wurden 3.194.000 Tonnen Al recycelt, 2020 werden es 7.700.000 Tonnen sein [7].

Beim Sammeln und Aufbereiten von Alu-Schrott geht es vorwiegend um Legierungen. Je sauberer der Schrott umso gefragter ist es für neue Verwendungszwecke.

\subsection{Magnesium Mg}

\subsubsection{Technische Daten}

Magnesium und seine Legierungen sind Werkstoffe mit hohem Anwendungspotential [8] für den Leichtbau. Dichte $1,74 \mathrm{~g} / \mathrm{cm}^{3}$.

Magnesium ist ca. $33 \%$ leichter und etwas weniger fest als Aluminium.

\subsubsection{Herstellung}

Die Herstellung erfolgt durch Schmelzflusselektrolyse analog derjenigen des Aluminium. Wichtige Legierungen sind: MgA116Zn und G-MgAl19Zn (AZ91) [8].

\subsubsection{Verarbeitung / Processing}

Magnesiumlegierungen lassen sich sehr gut im Druckgießen, Strangpressen oder Gesenkschmieden verarbeiten. Die Verarbeitung erfordert hohe technologische Ansprüche wegen der chemischen Reaktionsfreudigkeit: Mg-Späne können sich selbst entzünden. Mg-Schmelze muss unter Schutzgas verarbeitet werden.

\subsubsection{Eigenschaften}

Magnesium zeigt einige herausragende Eigenschaften, vor allem ein sehr gutes Verhältnis von Festigkeit und Gewicht $[9,10]$.

Allerdings sind die mechanischen Eigenschaften von Magnesium in seiner reinsten Form nur mäßig [11], und geringer im Vergleich zu Aluminium.

Magnesium bildet an der Luft eine weiße poröse Oxidschicht und ist nur bedingt witterungs- bzw. chemikalienbeständig.

Durch mäßige mechanische Eigenschaften wird es für technische Zwecke nur als Bestandteil von Legierungen verwendet.

Im Vergleich zu Al-Legierungen erreichen sie bei Raumtemperatur und erhöhter Temperatur nur geringe Festigkeitswerte [12].

Dem geringen Unterschied in der Dauerfestigkeit steht eine höhere Kerbempfindlichkeit entgegen.

Riefenfreie Oberflächen und Vermeidung von Kerben sind daher für die Anwendung von Mg-Legierungen unerlässlich [9].

Der niedrige Elastizitätsmodul macht $\mathrm{Mg}$-Legierungen unempfindlich gegen Schlag- und Stoßbeanspruchung.

Das extrem elektronegative Potential von Mg und seinen Legierungen macht einen Korrosionsschutz gegen Feuchtigkeit und Witterungseinflüsse erforderlich. 
Mg-Teile, die auch nur kurzzeitig einer aggressiven Atmosphäre ausgesetzt sind, müssen mit einer porenfreien Lackierung versehen werden.

Besonders ist darauf zu achten, dass bei Berührung mit anderen Werkstoffen Kontaktkorrosion vermieden wird, z.B. müssen Stahlschrauben verzinkt sein.

\subsubsection{Recycling / Recycling}

Der Stoffkreislauf von Magnesium [13] wird unter Beachtung bestimmter Schrottklassen 1 bis 9 klassifiziert.

Wegen der größeren Aktualität im leichten Automobilbau hat die TU Clausthal eine Arbeitsgruppe gebildet.

Magnesiumspäne sind wegen ihrer leichten Brennbarkeit ein tückisches Gefahrengut. Selbstverständlich ist die Entsorgung nach der BimSchV (Bundes Immissionsschutzverordnung) mittlerweile in einigen Betrieben problemlos möglich.

\subsection{Zink Zn}

\subsubsection{Technische Daten}

Zink kommt in verschiedenen Zink-Erzen vor.

Schmelzpunkt: $419^{\circ} \mathrm{C}$

Es kommt vor als:

- Zinkblende $(\mathrm{ZnS})$ ca. $67 \% \mathrm{Zn}$

- Zinkspat $\left(\mathrm{ZnCO}_{3}\right)$ ca. 52,1\% Zn

- Zinkoxyd ( $\mathrm{ZnO})$

Wegen der hohen Dichte ca. $7 \mathrm{~g} / \mathrm{cm}^{3}$ ist Zink erheblich schwerer als Aluminium mit 2,7 oder das leichte Magnesium mit 1,74. Für viele Produktionsbereiche ist es zu schwer.

\subsubsection{Herstellung}

Durch Flotation (Schwimmaufbereitung, Aufbereitungsverfahren zur Anreicherung von Mineralien etc.) aufbereitete Zn-Erze werden zur Befreiung von Schwefel und Kohlensäure geröstet. Bei ZnS wird der Schwefel zu Schwefeloxyd verbrannt und das Zinksulfid in Zinkoxid umgewandelt. Das metallische $\mathrm{Zn}$ wird entweder auf trockenem oder nassem Wege gewonnen.

\subsubsection{Verarbeitung}

Zink-Gusslegierungen werden im Sand-, Kokillen, Druck- und Spritzgießverfahren hergestellt. Sehr bekannte, relativ billige Zinkdruckgusslegierungen sind ZAMAKLegierungen

\subsubsection{Eigenschaften}

Zink ist unbeständig gegen Säuren, Salzlösungen und starke Laugen. Dennoch ist Zn witterungsbeständig, weil sich das Metall an feuchter Luft mit einer schützenden ZinkKarbonat-Schicht überzieht.

Im Vergleich zum gut span verarbeitenden $\mathrm{Mg}$ und $\mathrm{AL}$ ist das schlecht spanende $\mathrm{Zn}$ nicht gut $\mathrm{zu}$ bearbeiten, es schmiert.
Zn hat die größte Wärmeausdehnung aller Metalle, und ist viertbester elektrischer Leiter.

Wegen der chemischen Reaktivität darf Zn nicht mit Lebensmitteln in Kontakt kommen [9].

Im gewalzten Zustand hat Zink eine Zugfestigkeit von $200 \mathrm{~N} / \mathrm{mm}$ ، bei einer Bruchdehnung von etwa $20 \%$, jedoch neigt $\mathrm{Zn}$ bereits bei Raumtemperatur zum Kriechen (in Querrichtung weniger stark ausgeprägt).

Zn-Druckgussteile, meistens aus Legierungen von $\mathrm{Zn}$ und $\mathrm{Al}$ und $\mathrm{Cu}$, sind von hoher Maßgenauigkeit, jedoch empfindlicher gegen Korrosion als Reinzink.

\subsubsection{Recycling}

Zink bietet als Metall hervorragende Voraussetzungen, ohne Qualitätsverluste und mit geringem Energieaufwand beliebig oft wieder verwendet werden zu können. Die Recyclingqoute liegt bei über $80 \%$. Aus der Initiative Zink ist bekannt, dass jährlich etwa 2 Mio Tonnen $\mathrm{Zn}$ recycelt werden.

\section{Umweltbetrachtung}

Die Schonung der natürlichen Ressourcen, Nutzung der gespeicherten Energie nach Gebrauch, sind die Basis für das Recyclingverständnis der Industrie.

Dabei ist die geringe Sekundärenergieaufwendung beim Recycling z.B. bei Aluminium sehr interessant.

Sicher werden die Altwerte der Metalle, in Zukunft steigen. Eine beliebig oft $\mathrm{zu}$ wiederholende Anwendung macht Metalle zu Ressourcenbänken über Tage.

\subsection{Rio - Deklaration}

Der in der Rio-Deklaration festgelegte Grundsatz der „Nachhaltigkeit“" wird von der deutschen NE-Metallindustrie unterstützt:

Wirtschaft, Gesellschaft und Ökologie weltweit harmonisch zu entwickeln. Im Mittelpunkt der Klimadiskussion steht Kohlendioxid $\left(\mathrm{CO}_{2}\right)$.

Mögliche Reduktionspotentiale wurden bereits in der Vergangenheit von der deutschen NE-Industrie ausgeschöpft. Hierzu gehören :

Steigerung der Sekundärmetallerzeugung, Ener-gieeinsatzminimierung, Erhöhung der Energieeffizienz in der Verarbeitung, Ersatz $\mathrm{CO}_{2}$-reicher durch $\mathrm{CO}_{2}$-ärmere Brennstoffe.

Die erwähnte Selbstverpflichtungserklärung, die eine spezifische Energieeinsparung pro Tonne Produktion um $22 \%$ vorsieht, dient der noch weitgehenden Reduktion des $\mathrm{CO}_{2}$-Ausstoßes.

\subsection{Festlegung des Materiales der Lampenabdeckung}

Durch die eingehende Diskussion der zur Wahl stehenden NE-Metalle für die beiden. 
Druckdeckel wurde Aluminium bzw. eine Aluminiumlegierung ausgewählt.

Mit den beiden, parallel zur Polycarbonatabdeckung montierten Schutzprofilteilen, ebenfalls aus Aluminium sind gleiche Werkstoffe für den Rahmen der Lampenabdeckung gewählt worden.

Unter Berücksichtigung mit möglichst wenig unterschiedlichen und leicht recycelbaren Werkstoffen zu arbeiten, wurde der Aspekt der Produktverantwortung besonders berücksichtigt.

\subsection{Berücksichtigung des Längenausdehnungs- koeffizienten}

Da die Leuchtensysteme der Witterung und dabei differenzierten Umgebungstemperaturen ausgesetzt werden, mussten die unterschiedlichen Längenausdehnungskoeffizienten der beiden Werkstoffe, Polycarbonat unverstärkt, und Aluminium, für die Längenmaße der Lampenabdeckung beachtet werden.

Da das Grundprofil aus dem gleichen stranggepressten Aluminium besteht wie der Aluminiumrahmen der Abdeckung, war die Betrachtung nur für die Lampenabdeckung mit der Lichtaustrittsfläche aus klarem und schlagfesten Polycarbonat relevant. Je nach PC Werkstoffspezifikation schwankt der Wert Von 66 bis 70 .

Als Beispiel ist in diesem Artikel eine Länge der Abdeckung von $1.500 \mathrm{~mm}$ angegeben.

Eine Umrechung auf die unterschiedlichen Abmessungen der Lampenabdeckungen, bedingt durch die Längen der Leuchtmittel ist linear möglich.

Für zwei Einsatztemperaturbereiche wurden die Berechnungen vorgenommen.

Die Formel, Tabelle 1 für die Berechnung des Längenausdehnungskoeffizienten lautet:

Tabelle 1. Formel

Table 1. Formula

Wärmeausdehnung: $l(T)=l_{0}(1+\alpha \Delta T) \quad[\alpha]=K^{-1}$

In der nachstehenden Tabelle 2 sind die Längenausdehnungskoeffizienten für Aluminium und Polycarbonat angegeben. Die Formel in Tab. 1 und die Werte in Tabelle 2 sind Grundlage für die Berechnung und Anwendung in Tabelle 3 um spätere Eigenspannungen bei unterschiedlichen Umgebungstemperaturen im dem Endprodukt zu vermeiden.

Der Anwendungsbereich der Leuchten kann auch unter besonderen Bedingungen mit Zugluft und starken Temperaturunterschieden sein.z.B.

Übergang von geschützten Unterführungen in den Freibereich.

Länge $(1)=1,5 \mathrm{~m}=1500 \mathrm{~mm}$

BITTE ALLE ANGABEN in mm und Quellenhinweis neu 14

(Entschuldigung )
Tabelle 2. Beispiele für $\alpha^{1}$

Table 2. Examples for $\alpha^{1}$

\begin{tabular}{|c|c|c|}
\hline Material & $\alpha / 10^{-6} K^{-1}$ & $\alpha / K^{-1}$ \\
\hline Aluminium & 23.0 & 0.000023 \\
\hline Polycarbonat (PC) & 66 bis 70 & 0.000066 bis 0.000070 \\
\hline
\end{tabular}

Tabelle 3. Anwendung

Table 3. Application

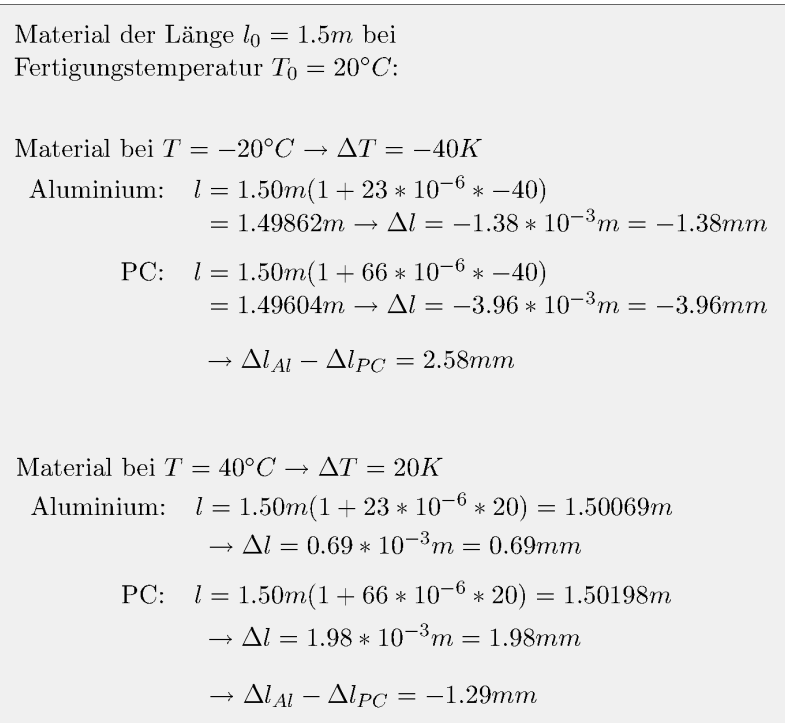

In der Tabelle 3 wurden die Berechnungen für die spätere Anwendung der beiden Werkstoffe Aluminium und Polycarbonate durchgeführt.

Das Ergebnisse zeigt eine Differenz von 1,29 mm bzw. 2,58 mm bei einem anderen Delta - T, unter der Prämisse wenn die Nachschwindung der Werkstoffe nach dem Verarbeitungsprozess abgeschlossen ist.

Die Lichtaustrittsfläche besteht aus Polycarbonat und wird aus Schutzgründen in die Aluminiumschutzprofilteile, Abb 3, (6) und stirnseitig von den beiden Druckdeckeln, Abb 3, (3) umschlossen.

Bei einer Nichtberücksichtigung der unterschiedlichen Längenausdehnungskoeffizienten wäre als Folge, bei gleichen Grundlängen, dass die Stirndruckdeckel, bei ansteigenden Temperaturen weggedrückt, und bei niedrigeren Temperaturen ein Spalt entstehen würde .

Folge: die Leuchte öffnet sich, Feuchtigkeit, Staub, Insekten etc. dringen in das Leuchtengehäuse ein.

Gleichzeitig entspricht die Leuchte nicht mehr den Prüfnormen.

Formeln und Längenausdehnungskoeffizienten sind aus den Quellen der Tabelle 4 entnommen.

Tabelle 4. Quellen

Table 4. Sources

http://infos.aus-germanien.de/W\%C3\%A4rmeausdehnungskoeffizient http://www.goodfellow.com/csp/active/STATIC/G/Polycarbonat.HTML 


\section{Fazit}

In diesem Artikel wurde die Neuentwicklung einer Leuchtenserie für den Einsatz für besonders durch Vandalismus gefährdeten Umgebungen erörtert.

Die wichtigsten Konstruktionsparameter unter dem Aspekt der Produktverantwortung waren:

- extrem schlagfest

- extrem flach, mit wenig Angriffsflächen

- Langlebigkeit der eingesetzten Werkstoffe bei Schlageinwirkung

- sehr gute Recyclingmöglichkeiten

- Lampenabdeckung resistent gegen Sprühlacke (Graffiti)

Unter Berücksichtigung des Projektmanagements für das gesamte Projekt konnten Fehler und Kontrolle erheblich verbessert werden.

Insbesondere die Terminabstimmung und der interdisziplinäre Bereich wurden durch die Projektleitung überwacht und erfolgreich gesteuert.

Vor der Freigabe der Werkzeuge konnten weiter die Fehlerhaftigkeit bzw. Analyse durch das Rapid Prototyping gesenkt werden.

Ein anderer Vorteil bei der Konstruktion war die CAD Anwendung im 3 D-Verfahren. Das heute bereits zur selbstverständlich geworden ist.

Der Beitrag zeigt außerdem wie intensiv die Auseinandersetzung mit der geeigneten Werkstoffwahl, insbesondere für die Gehäuseendkappe gewesen ist.

Für die transparente Lichtaustrittsfläche der Lampenabdeckung ist eine Alternativlösung in modifizierter Materialausführung möglich. Die Oberfläche wird dadurch dauerhaft geschützt gegen Grafittispraylacke. Im CoExtrusionsverfahren wird eine dünne Oberfläche über das Polycarbonat im Fertigungsprozess angebracht. Die Schlagfestigkeit wird dadurch nicht beeinträchtigt.

Diese Variante ist der einer nachträglich aufgebrachten Schutzlackierung vorzuziehen.

Bei der Beurteilung und der interdisziplinären Begutachtung der Stereolithographieteile wurde erkannt, dass die Dichtungsgeometrie der Lampenabdeckung zum Leuchtengehäuse im Bereich der Fassungsträgerendkappe technisch verändert werden musste.

Diese Erkenntnis war für die spätere Schutzartprüfung, strahlwassergeschützt und staubdicht, außerordentlich wichtig. ( Schutzart IP 65 )

Die Endkappen aus einer kupferfreien Aluminiumlegierung z.B. Al 230, AlSi10Mg, werden aus Qualitätsgründen, nach dem Fertigungsprozess phosphatiert/gebeizt und anschließend gepulvert, um evt. spätere Lunker bzw. Oberflächenprobleme durch Entgasung sofort festzustellen und zu sortieren.

Die seitlichen Schutzprofilteile (6) und das Grundprofil
(1) werden nach dem Strangpressen, aneloxiert mit einer Stärke von ca. $8 \mu \mathrm{m}$.

Nach alledem ist eine Leuchte entstanden, die hervorragende technische Merkmale aufweist.

Die gelungene Optik und die haptischen Eigenschaften wird den Anwendungsbereich der Neuentwicklung auch für Gebäude mit besonderer Architektur attraktiv machen.

Darüber hinaus wird der Produktverantwortung und der späteren Recycelbarkeit große Aufmerksamkeit gewidmet.

Dies zeigt sich in der ausführlichen Diskussion zur Wahl der Werkstoffe, bei der man sich schließlich auf wenige leicht recycelbare Materialien beschränkte.

Für das Basisgrundgehäuse, und für den Lampenabdeckungsrahmen mit Endkappe wurde Aluminium verwendet.

Durch das intelligente, technische Leuchtenkonzept ist eine Lichtbandanwendung in Verbindung mit einem optisch angepassten, vandalismusgeschützten Medienkanal eine weitere Lösungsvariante und bietet dem Betreiber mit denselben Ersatzteilen weitere Einsatzmöglichkeiten.

Unter der Beachtung der Wirtschaftlichkeitsanalyse [2] in der Projektplanung ist auch ein ökonomisch interessantes Produkt mit bestem Preis- Leistungsverhältnis entstanden.

Vor Produkteinführung sind nun alle beteiligten Abteilungen, Produktion, Materialwirtschaft, Vertrieb, Marketing mit der Projektleitung und dem Entwicklungsteam gefordert und eine erfolgreiche Markteinführung zu gewährleisten.

\section{Literatur}

1. Gebhard Andreas, Rapid Prototyping, Werkzeuge für die schnelle Produktentstehung, Carl Hanser Verlag München Wien, 2 völlig überarbeitete Auflage, 2000.

2. Ehrlenspiel Klaus, Integrierte Produktentwicklung, Denkabläufe Methodeneinsatz Zusammenarbeit, Carl Hanser Verlag, 2, überarbeitete Auflage, 2003.

3. Ginevicius Romualdas, Hausmann Thomas Schlomo Schafir, Projektmangement - Einführung, Deutscher Betriebswirte Verlag, Gernsbach 2005-03-19.

4. Kreislaufwirtschafts- und Abfallgesetz ist seit 07.Oktober.1996 in Kraft. Gesetz zur Förderung der Kreislaufwirtschaft und Sicherung der umweltverträglichen Beseitigung von Abfällen. Abkürzung : KrW-AbfG.

5. Ebel Bernd, Produktionswirtschaft, 8. völlig neue Auflage 2003 , Friedrich Kiel Verlag Ludwigshafen am Rhein.

6. Recycling-Magazin Sonderdruck 17/2002, Aluminium, Zum Recycling beonders geeignet.

7. Aluch, Aluminum Verand Schweiz, Fachartikel über Aluminium Recycling.

8. Hering Ekbert, Modler Karl-Heinz, Grundwissen des Ingenieur, Fachbuchverlag Leipzig, 2002.

9. Bergmann Wolfgang, Werkstofftechnik 1, Struktureller Aufbau von Werkstoffen- Metallische Werkstoffe - Polymerwerkstoffe - Nichtmetallisch - anorganische Werkstoffe, Carl Hanser Verlag München Wien, 4 Auflage, 2002. 
10. Seidel Wolfgang, Werkstofftechnik, Werkstoffe -Eigenschaften- Prüfung-Anwendung, Carl Hanser Verlag München Wien, 5 Auflage, 2003.

11. Stattmann Nicola, Materialtechnologie Handbuch, Hrsg. Rat für Formgebung, Ludwigsburg av-Ed. 2000.
12. Beiz, W.; Grote, K.-H. Dubbel Taschenbuch für den Maschinenbau, 20 Auflage, Springer Verlag Berlin Heidelberg New York.

13. Ditze, A. Stroffkreislauf von Magnesium. Aluminium 75, No 3 1999, S. 207-220.

\section{VANDALIZMUI ATSPARIŲ APŠVIETIMO SISTEMŲ KONSTRUKCINIAI SPRENDIMAI}

\section{F. Pracht}

Santrauka. Dažnai visuomeninius pastatus ir viešojo transporto priemones niokoja vandalai. Jų objektu tampa ir apšvietimo sistemos. Šiame straipsnyje aptarta naujos ir saugios (turinčios I ir II atsparumo klases) apšvietimo sistemos gamyba, ivvertinta jos pakartotinio naudojimo galimybè ir galimybė naudoti ne tokias taršias medžiagas.

Raktažodžiai: atsparumas mechaniniam poveikiui, ilgaamžiškumas, pakartotinis naudojimas, projektų vadyba, atsparumas atmosferos poveikiui, vandalizmas, patogumas eksploatuoti, tempimo stiprumo riba.

Friedhelm PRACHT. Managing Director of the pracht group $\subset$, international manufacturer of waterproof luminaires systems for application Areas and plastics.

First degree engineer in mechanical engineering closed successful (1975) University of Apllied Sciences Giessen and a learned manufacturer of metallic moulds (1969). Postgraduate study in oeconomics (1975 - 1977) of the Justus-Liebig University, Giessen. Occupation after the study. After the sudden death of his father and company founder Alfred Pracht he stepped into the Alfred Pracht KG which had already been founded by his parents in (1963).

For twenty-five years he successfully conducts today’s pracht group $\bigodot$ in Germany. Responsible of Research and Development of the Company.

As a doctoral candidate of engineering technology on the Vilnius Gediminas Technical University, in his dissertation he deals with the "constructional optimization of luminaire systems for special application areas in accordance with the product responsibility as decreed in $\S 22$ of the Kreislaufwirtschafts- und Abfallgesetz (Law of Recycling Management and Waste Management Law)”.

Author of about 14 scientific articles.

Research interests: constructional optimation and materials of luminaire systems for special apllication, optimation of processing duroplastics and thermoplastics, development - research and efficiency from waterproof luminaire in use at very low temperatures, integration productpolitic and according with the product responsibility. 